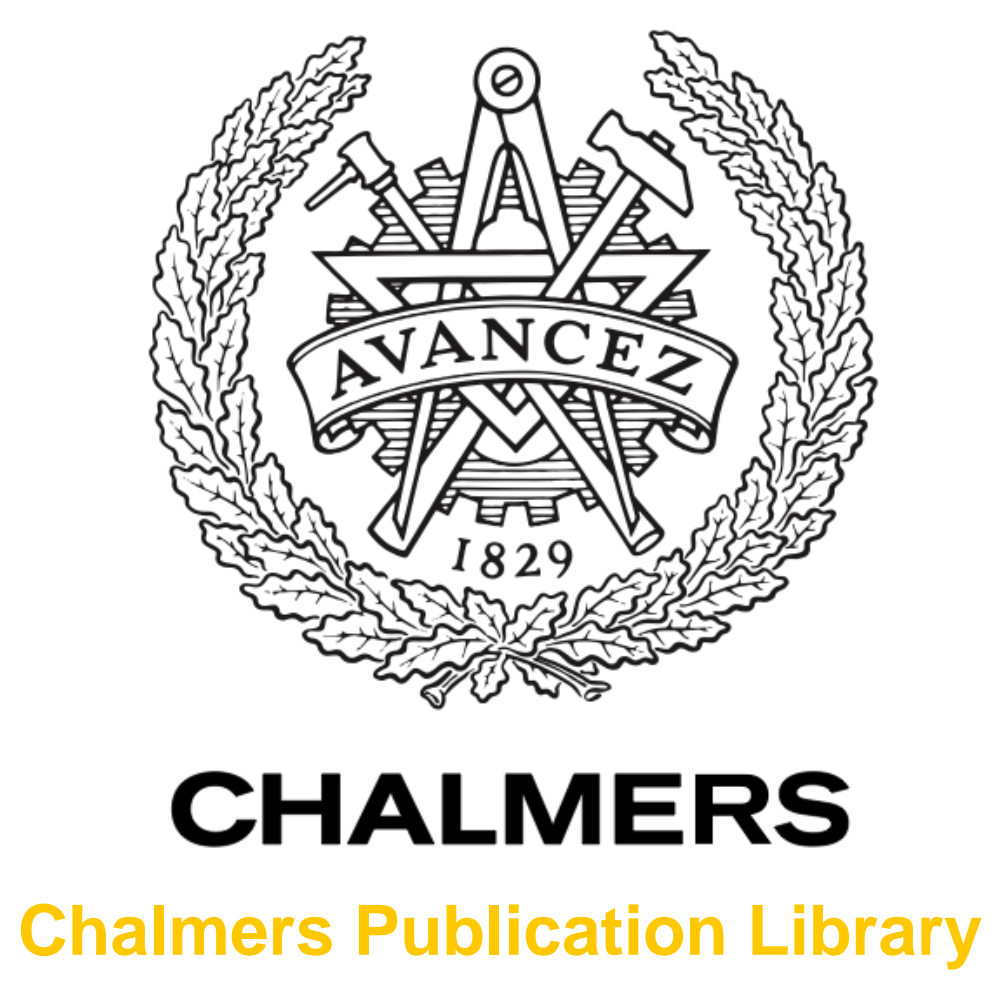

\title{
Unusual thermopower of inhomogeneous graphene grown by chemical vapor deposition
}

This document has been downloaded from Chalmers Publication Library (CPL). It is the author's version of a work that was accepted for publication in:

\section{Applied Physics Letters (ISSN: 0003-6951)}

Citation for the published paper:

Nam, Y. ; Sun, J. ; Lindvall, N. (2014) "Unusual thermopower of inhomogeneous graphene grown by chemical vapor deposition". Applied Physics Letters, vol. 104(2), pp. 021902.

http://dx.doi.org/10.1063/1.4861745

Downloaded from: http://publications.lib.chalmers.se/publication/192584

Notice: Changes introduced as a result of publishing processes such as copy-editing and formatting may not be reflected in this document. For a definitive version of this work, please refer to the published source. Please note that access to the published version might require a subscription. 


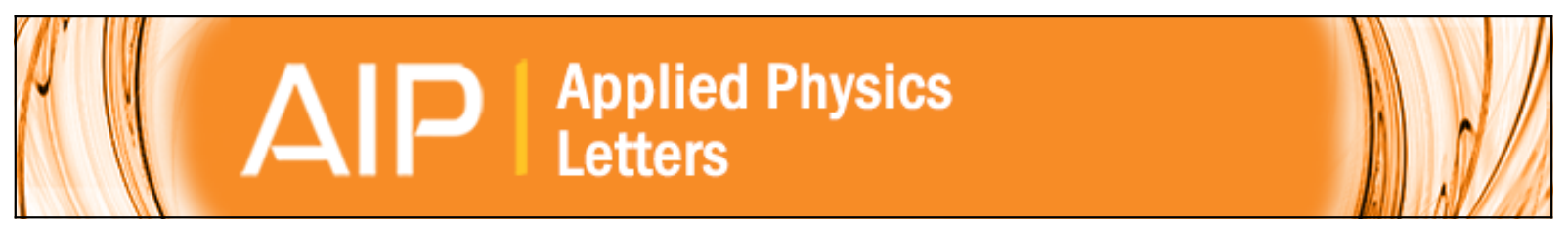

\section{Unusual thermopower of inhomogeneous graphene grown by chemical vapor} deposition

Youngwoo Nam, Jie Sun, Niclas Lindvall, Seung Jae Yang, Chong Rae Park, Yung Woo Park, and August Yurgens

Citation: Applied Physics Letters 104, 021902 (2014); doi: 10.1063/1.4861745

View online: http://dx.doi.org/10.1063/1.4861745

View Table of Contents: http://scitation.aip.org/content/aip/journal/apl/104/2?ver=pdfcov

Published by the AIP Publishing

$\stackrel{A}{A} \mathbb{P} P$ Re-register for Table of Content Alerts

Create a profile.

Sign up today! 


\title{
Unusual thermopower of inhomogeneous graphene grown by chemical vapor deposition
}

\author{
Youngwoo Nam, ${ }^{1,2, a)}$ Jie Sun, ${ }^{2}$ Niclas Lindvall, ${ }^{2}$ Seung Jae Yang, ${ }^{3}$ Chong Rae Park, ${ }^{3}$ \\ Yung Woo Park, ${ }^{1}$ and August Yurgens ${ }^{2}$ \\ ${ }^{1}$ Department of Physics and Astronomy, Seoul National University, Seoul 151-747, South Korea \\ ${ }^{2}$ Department of Microtechnology and Nanoscience, Chalmers University of Technology, \\ SE-412 96 Gothenburg, Sweden \\ ${ }^{3}$ Department of Materials Science and Engineering, Seoul National University, Seoul 151-747, South Korea
}

(Received 30 October 2013; accepted 27 December 2013; published online 13 January 2014)

\begin{abstract}
We report on thermopower (TEP) and resistance measurements of inhomogeneous graphene grown by chemical vapor deposition (CVD). Unlike the conventional resistance of pristine graphene, the gate-dependent TEP shows a large electron-hole asymmetry. This can be accounted for by inhomogeneity of the CVD-graphene where individual graphene regions contribute with different TEPs. At the high magnetic field and low temperature, the TEP has large fluctuations near the Dirac point associated with the disorder in the CVD-graphene. TEP measurements reveal additional characteristics of CVD-graphene, which are difficult to obtain from the measurement of resistance alone. (C) 2014 AIP Publishing LLC. [http://dx.doi.org/10.1063/1.4861745]
\end{abstract}

Thermopower (TEP) is a useful tool in studies of intrinsic conduction mechanism in graphene, complementing resistivity measurements. TEP (also called the Seebeck coefficient $S$ ) is a measure of the electric field $E$ retarding diffusion of charge carriers in response to the temperature gradient $\nabla T$ in the open-circuit condition, $E=S \nabla T$. In general, the sign of TEP is decided by the type of majority carriers, i.e., $S>0$ for holes and $S<0$ for electrons.

Graphene has a unique band structure with the gapless conical electron- and hole bands. ${ }^{1}$ To date, TEP of the single-layer graphene has been extensively investigated both theoretically ${ }^{2}$ and experimentally. ${ }^{3-6}$ In experiment, high quality graphene samples obtained by mechanical exfoliation from graphite are used in most cases, to minimize the influence of defects and impurities and elucidate fundamental thermoelectric transport mechanism. The reported TEP is usually in the range of $\leq 100 \mu \mathrm{V} / \mathrm{K}$ and is generally analyzed in terms of semi-classical Mott relation.,

Meanwhile, the TEP measurements on graphene grown by chemical vapor deposition (CVD) have largely been focused on application aspects. For example, gas-flow sensors, ${ }^{7}$ surface charge doping indicators ${ }^{8}$ were demonstrated by employing atomically small thickness of graphene and sensitivity of its TEP to the variation of charge carrier density and type. Oxygen plasma treatment of few layer CVDgraphene allowed obtaining high TEP $(\approx 700 \mu \mathrm{V} / \mathrm{K})$, which can be promising for energy harvesting (heat-to-electricity conversion). ${ }^{9}$ Recently, a metallic temperature dependence of the TEP in few layer CVD-graphene was reported. ${ }^{10} \mathrm{By}$ virtue of CVD method capable of producing large-area graphene, the abovementioned TEP measurements on CVDgraphene were performed in a conventional way, using wire thermocouples and chip-resistor heaters; all this without undergoing painstaking microfabrication process. The millimeter sized CVD-graphene is usually transferred onto

\footnotetext{
${ }^{\text {a) }}$ Author to whom correspondence should be addressed. Electronic mail: youngwoo.nam@chalmers.se
}

insulating substrates (such as glass and quartz) and TEP of the whole graphene area is measured without taking care of selecting a clean area, controlling charge carrier density, or applying the magnetic field. However, the large-area CVDgraphene usually has many microscopic defects such as wrinkles and domain boundaries generated during growth/transfer process, all much likely affecting TEP. To address the role of inhomogeneity and structural defects in TEP of graphene, it is necessary to "zoom-in" into micro scale, similarly to the previous TEP measurements on the exfoliated graphene.

Here, we report on TEP of inhomogeneous CVDgraphene as a function of the charge carrier density $(n)$, temperature $(T)$, and magnetic field $(B)$ for both the longitudinal $\left(S_{\mathrm{xx}}\right)$ - and transverse $\left(S_{\mathrm{xy}}\right)$ directions with respect to the temperature gradient. Interestingly, we observe a significant electron-hole point asymmetry in the TEP measurements, which is not seen in the resistance data. This behavior can be attributed to individual graphene regions having different TEPs in the inhomogeneous sample. At the high magnetic field and low temperature, the TEP has large fluctuations near the Dirac point associated with the insulating $\nu=0$ quantum Hall state resulting from the disorder-induced energy gap opening at the central Landau level (LL) $(\nu=0)$. On the other hand, the Nernst signal $\left(S_{\mathrm{xy}}\right)$ shows such fluctuations only for electron-like carriers.

Large area graphene is synthesized by CVD on copper foils ${ }^{11}$ and transferred onto a Si substrate covered by $300 \mathrm{~nm}$ $\mathrm{SiO}_{2}$. Rectangular shaped graphene Hall bar $(10 \mu \mathrm{m} \times 50 \mu \mathrm{m})$ is made using the optically clean graphene regions without holes and residues. Subsequently, heaters, temperature sensors, and electrical leads are defined from the thermally evaporated $\mathrm{Au} / \mathrm{Ti}$ thin films $(60 \mathrm{~nm} / 5 \mathrm{~nm})$ (see the upper left inset in Figure 1(a)). We mainly measure the steady state TEP (a low frequency ac TEP method have also been used to confirm the reliability of measurements) in the linear regime ( $\Delta T \ll T$ ) while simultaneously measuring the four-probe ac resistance using a low-frequency lock-in technique. ${ }^{12}$ 
The upper left inset in Figure 1(a) shows an optical image of the TEP device where graphene boundaries are marked by the white solid-line rectangular in this picture. Micro-scale heaters and temperature sensors are placed at the both sides of the graphene having also four standard electrical contacts. The middle part of graphene marked by yellow dashed line is characterized using AFM (Figure 1(b)) and Raman D-band mapping (Figure 1(c)). In the AFM image, we observe wrinkles in the diagonal direction. Such wrinkles are quite common for CVD-graphene. Much likely, they appear during the transfer process. The Raman D-band mapping yields a similar image since the D-band peak intensity indicates defect sites and grain boundaries in graphene. ${ }^{13}$ Figure 1(d) shows two Raman spectra corresponding to two different sites: A (on the wrinkle) and B (outside the wrinkle). In contrast to flat region $\mathrm{B}$, "wrinkled" region A has both a much stronger intensity of the D-band $\left(\approx 1350 \mathrm{~cm}^{-1}\right)$ and additional weak $\mathrm{D}^{\prime}$-band $\left(\approx 1620 \mathrm{~cm}^{-1}\right)$. It is known that the $\mathrm{D}^{\prime}$-band is observed in the highly defective graphene undergoing intentional oxidization, hydrogenation, and fluorination. ${ }^{14,15}$ It is reasonable that the graphene wrinkle is more disordered than the flat region. The ratio of $I_{2 \mathrm{D}}$ to $I_{\mathrm{G}}$ is also diminished to $\approx 1.8$ at region A compared to the flat region $\mathrm{B}\left(I_{2 \mathrm{D}} / I_{\mathrm{G}} \approx 3.8\right)$.

We measure the resistance while tuning the gate voltage for various temperatures (see Figure 1(a)). The resistance has a maximum at the Dirac point, $V_{\mathrm{DP}} \approx 7.7 \mathrm{~V}$. The estimated mobility, $\mu \approx 650 \mathrm{~cm}^{2} / \mathrm{Vs}$, at $T=150 \mathrm{~K}$ is quite low. The resistance shows insulating behavior $(d R / d T<0)$ in the whole range of the gate voltage (i.e., irrespective of charge carrier density). The insulating behavior is consistent with the previous results on defective exfoliated graphene ${ }^{14,16}$ and CVD-graphene at low carrier density where thermal activation of charge carriers across inhomogeneous electron-hole puddles dominates. ${ }^{17}$
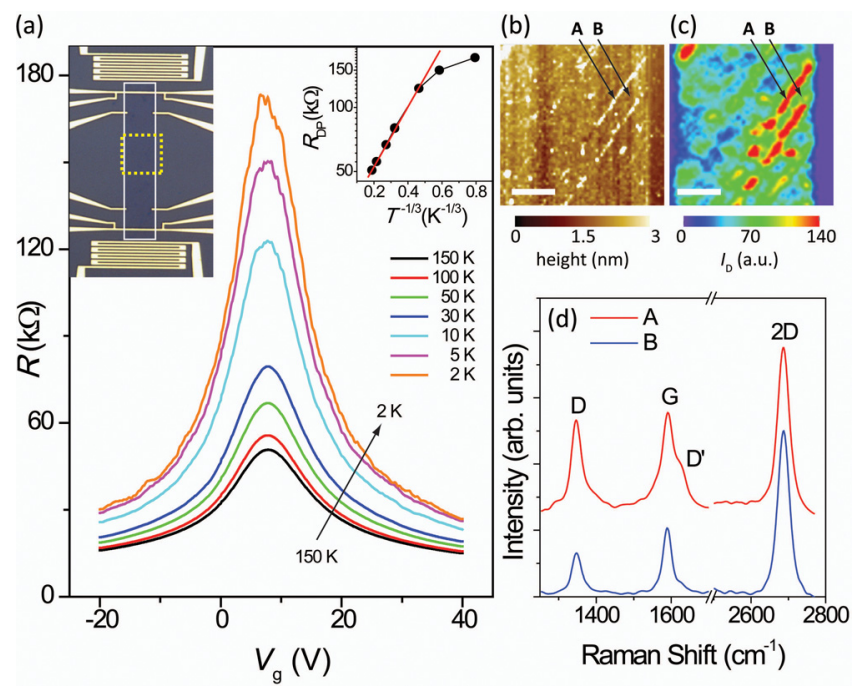

FIG. 1. (a) The resistance versus back gate voltage $\left(V_{\mathrm{g}}\right)$ at various temperatures. The upper left inset displays an optical image of the device where graphene boundaries $(10 \mu \mathrm{m} \times 50 \mu \mathrm{m})$ are marked by the white rectangle. The upper right inset indicates the resistance maximum at the Dirac point $\left(R_{\mathrm{DP}}\right)$ as a function of temperature. Images of (b) AFM- and (c) Raman mapping of D-band intensity $\left(I_{\mathrm{D}}\right)$ correspond to the same graphene region enclosed by yellow dashed line in the upper left inset of (a). Both scale bars are $3 \mu \mathrm{m}$. (d) The Raman spectra of graphene at two sites: A (on the wrinkle) and B (outside the wrinkle) denoted by arrows in (b) and (c). Both curves are normalized to 2D-band intensity. Spectrum A is shifted upwards for clarity.
The upper right inset indicates the resistance maximum at the Dirac point $\left(R_{\mathrm{DP}}\right)$ as a function of temperature. For $T>10 \mathrm{~K}$, we can see that $R_{\mathrm{DP}}(T)$ can be explained by the heterogeneous model of two-dimensional variable-range hopping $\left(\ln R \propto T^{-1 / 3}\right)$ describing conduction mechanism of the system. In this model, conducting regions (ordered graphene) are separated by thin insulating regions (disordered graphene) allowing for tunneling between the conducting regions. ${ }^{18}$ We note that this model also agrees with our results of AFM- and Raman-spectra mapping, which reveal microscopic-scale inhomogeneity of our sample.

The sign of TEP corresponds to the type of majority carrier in the system and its magnitude is proportional to the average entropy transported by the charge carriers. TEP of graphene is positive (negative) when majority carriers are holes (electrons) and approaches zero in the high carrierdensity regime (far from the Dirac point) because the entropy per carrier decreases in a degenerate state. Near the Dirac point, TEP is zero since there are equal amounts of both types of carriers cancelling retarding electric field. Therefore, TEP of graphene should be an odd-function of the gate voltage with respect to the Dirac point (be point-symmetric).

First, we measure TEP $\left(S_{\mathrm{xx}}\right)$ of our sample at various temperatures in the absence of magnetic field (Figure 2(a)). TEP shows the expected odd-function tendency at high temperature $(T=150 \mathrm{~K})$ but becomes asymmetrically distorted with decreasing temperature. The zero crossing point of $S_{\mathrm{xx}}$ shifts to negative voltage away from the Dirac point (obtained from the $R\left(V_{\mathrm{g}}\right)$ curves in Figure 1(a)). At low temperature ( $T=50 \mathrm{~K}), S_{\mathrm{xx}}$ even becomes positive on the electron band side, also accompanied by fluctuations $\left(V_{\mathrm{g}}>20 \mathrm{~V}\right)$.

Disregarding phonon-drag effect TEP can be expressed as

$$
S_{x x}=-\frac{1}{e T}\left\{\frac{\int\left[\varepsilon-\varepsilon_{F}\right] \sigma^{\prime}(\varepsilon)[-\partial f / \partial \varepsilon] d \varepsilon}{\int \sigma^{\prime}(\varepsilon)[-\partial f / \partial \varepsilon] d \varepsilon}\right\}=-\frac{\left\langle\varepsilon-\varepsilon_{F}\right\rangle}{e T},
$$

where $\sigma^{\prime}(\varepsilon)\left(=e^{2} D \tau v_{\mathrm{F}}^{2} / 2\right)$ is the energy-dependent part of the total conductivity $\sigma\left(=\sigma=\int \sigma^{\prime}(\varepsilon)[-\partial f / \partial \varepsilon] d \varepsilon\right)$. Here, $e, D, \tau$, $v_{\mathrm{F}}, f$, and $\varepsilon_{\mathrm{F}}$ are electron charge, the density of states, momentum relaxation time, Fermi velocity, Fermi-Dirac distribution function, and Fermi energy, respectively. $\left\langle\varepsilon-\varepsilon_{\mathrm{F}}\right\rangle$ means averaged energy (relative to the Fermi energy), which is weighted by the partial conductivity near Fermi energy, $\sigma^{\prime}(\varepsilon)(-\partial f / \partial \varepsilon)$.

When the Fermi energy is near the Dirac point, TEP has contributions from both the electron- and hole bands. Due to unknown distribution of the electron-hole puddles and inherent inhomogeneity of CVD-graphene, the net TEP can have intricate dependence on the gate voltage and temperature. For instance, if the sample was composed of different types of graphene regions in series, the total effective TEP would be given by $S_{\text {eff }}\left(V_{g}\right)=\sum_{i} S_{i}\left(V_{g}\right) \Delta T_{i} / \sum_{i} \Delta T_{i}$, where $S_{i}$ and $\Delta T_{i}$ are the TEP of and the temperature difference across each graphene region, respectively. It is clear from this equation that it is enough to have some of the regions not responding to the gate voltage $\left(S_{j}\left(V_{\mathrm{g}}\right)=\right.$ const $)$ to break the symmetry of the generic $S\left(V_{\mathrm{g}}\right)$ for graphene relative to the point of origin $S=0, V_{\mathrm{g}}-V_{\mathrm{DP}}=0$. This differs from the 

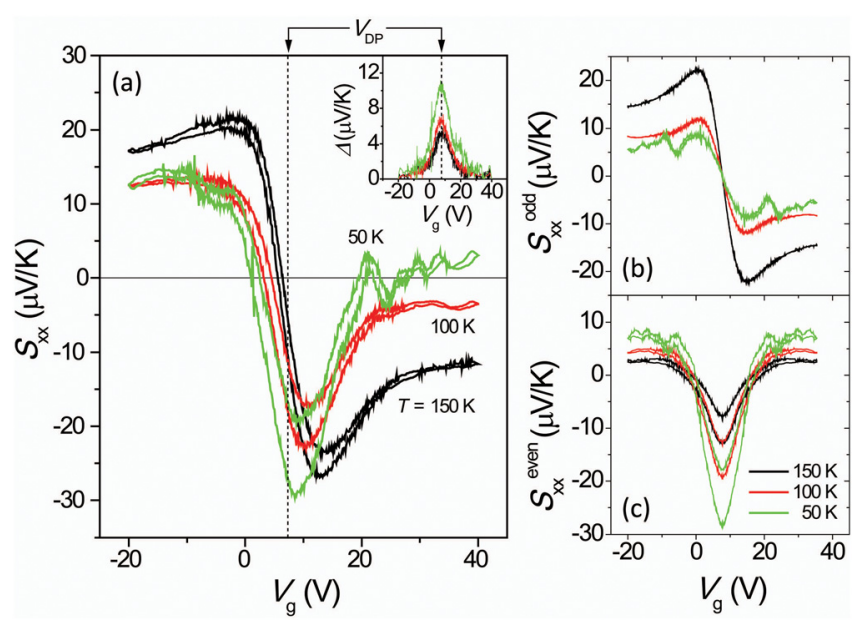

FIG. 2. (a) Longitudinal TEP $\left(S_{\mathrm{xx}}\right)$ versus gate voltage $V_{\mathrm{g}}$ for $T=150,100$, and $50 \mathrm{~K}$ in zero magnetic field. In the inset, $\Delta$ represents the difference of $S_{\mathrm{xx}}$ between positive- and negative gate voltage sweeps, which has the peak at $V_{\mathrm{DP}}$. Here, the two vertical dashed lines indicate the Dirac point, $V_{\mathrm{DP}}$ $(\approx 7.7 \mathrm{~V})$ corresponding to the peak position of $R\left(V_{\mathrm{g}}\right)$ in Figure 1(a). (b) Odd- and (c) even-components of $S_{\mathrm{Xx}}$ are extracted from (a) with respect to $V_{\mathrm{g}}=V_{\mathrm{DP}}$, and are denoted as $S_{\mathrm{xx}}{ }^{\text {odd }}$ and $S_{\mathrm{xx}}{ }^{\text {even }}$, respectively.

total resistance $R\left(V_{\mathrm{g}}\right)$ which in the abovementioned case would just acquire an offset while not breaking the symmetry relative to the vertical line $V_{\mathrm{g}}-V_{\mathrm{DP}}=0$. We believe that the presence of inhomogeneity explains the observed point-asymmetric behavior of TEP in our sample.

As seen in Figure 2(a), a hysteresis between different voltage sweep directions is observed for all temperatures. The hysteresis is largest near the Dirac point. The difference of $S_{\mathrm{xx}}$ between positive- and negative gate sweep directions (defined as $\Delta$ in the inset of Figure 2(a)) results in the even function having peak precisely at $V_{\mathrm{DP}}$. We assume that it somehow reflects the influence of the Dirac point on TEP result, although the measured $S_{\mathrm{xx}}$ itself does not directly manifest it owing to the non-zero value at $V_{\mathrm{DP}}$ and asymmetric behavior.

We separate $S_{\mathrm{xx}}\left(V_{\mathrm{g}}\right)$ into the odd- and even-components with respect to $V_{\mathrm{g}}=V_{\mathrm{DP}}$. The extracted odd-component $\left(S_{\mathrm{xx}}{ }^{\text {odd }}\right)$ is independent of the gate sweep direction, whereas the even-component $\left(S_{\mathrm{Xx}}{ }^{\text {even }} \pm \Delta / 2\right)$ contains hysteresis arising from $\Delta\left(V_{\mathrm{g}}\right)$, which is also even function. Figures 2(b) and 2 (c) display $S_{\mathrm{xx}}$ odd and $S_{\mathrm{xx}}$ even, respectively. We can see a strong negative deep in $S_{\mathrm{xx}}^{\text {even }}$ which is comparable with $S_{\mathrm{xx}}$ odd $S_{\mathrm{xx}}$ even increases as the temperature decreases, while $S_{\mathrm{xx}}$ odd decreases.

We note that the asymmetric TEP and mismatch between the gate voltages corresponding to the maximum of resistance $\left(V_{\mathrm{g}}=V_{\mathrm{DP}}\right)$ and zero of TEP is observed for several low mobility $\left(\mu<1000 \mathrm{~cm}^{2} / \mathrm{Vs}\right) \mathrm{CVD}$-graphene samples. We also confirm this for the faster ac TEP measurements, where the hysteresis effect is nevertheless smaller. In addition, we have verified that the mismatch is not seen for the high mobility CVD-graphene $\left(\mu \approx 3000 \mathrm{~cm}^{2} / \mathrm{Vs}\right)$, which is consistent with the TEP of exfoliated graphene. . $^{3,4}$

To simulate the inhomogeneity effect on $R\left(V_{\mathrm{g}}\right)$ and $S_{\mathrm{xx}}\left(V_{\mathrm{g}}\right)$, we assume a simple lumped-element network (see Figure 3(a)). Each segment has its own resistance and contains a thermoelectric voltage source. Here, two different
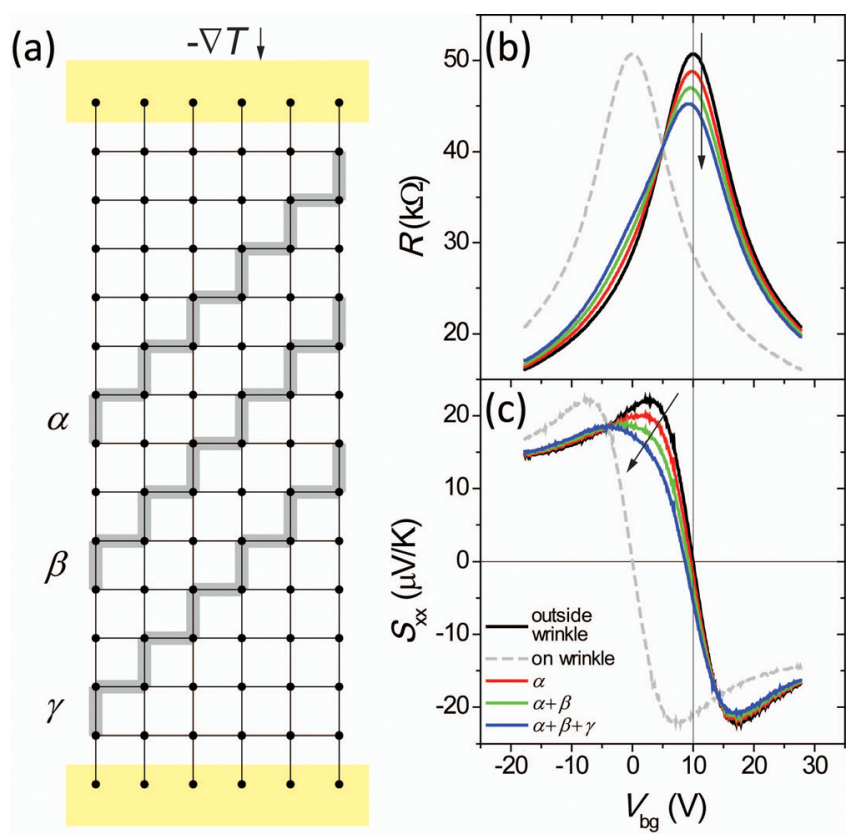

FIG. 3. (a) Simulation grid modeling inhomogeneous graphene. Each segment connecting two points represents individual graphene region outside the wrinkle (thin lines) or on the wrinkle (zigzag lines with grey shadows labeled by $\alpha, \beta$, and $\gamma$ ). Yellow rectangular bars at both ends display metal contacts. (b) Total resistance $R\left(V_{\mathrm{g}}\right)$ - and (c) thermopower $S_{\mathrm{xx}}\left(V_{\mathrm{g}}\right)$ of the model for increasing number of wrinkles as indicated by the arrows.

types of segments correspond to graphene region outside the wrinkle (thin lines) and on the wrinkle (zigzag lines with grey shadows). The corresponding $R\left(V_{\mathrm{g}}\right)$ and $S_{\mathrm{xx}}\left(V_{\mathrm{g}}\right)$ for the two regions are shown as black solid- and grey dashed line in Figures 3(b) and 3(c), respectively. We set the Dirac points at 10 and $0 \mathrm{~V}$ for these regions.

Under these conditions, we follow the variation of the total resistance $R\left(V_{\mathrm{g}}\right)$ and thermopower $S_{\mathrm{xx}}\left(V_{\mathrm{g}}\right)$ as the number of wrinkles changes, by solving a system of Kirchhoff's equations. We can see that $S_{\mathrm{xx}}\left(V_{\mathrm{g}}\right)$ becomes more distorted and asymmetric with increasing the number of wrinkles compared to $R\left(V_{\mathrm{g}}\right)$. The increase of wrinkle length or width also results in similar behavior. This simplified model enables us to qualitatively demonstrate the sensitivity of TEP to sample inhomogeneity as observed in our experiments, as also has very recently been shown by using local TEP measurements. ${ }^{19}$

We now consider the magneto-resistance and magnetoTEP in the magnetic field of $13 \mathrm{~T}$ as functions of the gate voltage for both the longitudinal and transverse directions with respect to the bias-current- and temperature-gradient directions. For convenience, the gate voltage $V_{\mathrm{g}}{ }^{\prime}$ is measured relative to $V_{\mathrm{DP}}$, i.e., $V_{\mathrm{g}}{ }^{\prime}=V_{\mathrm{g}}-V_{\mathrm{DP}}$. The corresponding filling factors $\nu\left(=h n / e B=h \beta V_{\mathrm{g}}^{\prime} / e B\right)$ are marked in the upper $\mathrm{x}$-axis of plots in Figure 4 , where $h, e$, and $\beta$ $\left(\sim 7.2 \times 10^{10} \mathrm{~cm}^{-2} \mathrm{~V}^{-1}\right)$ are Plank's constant, the electron charge, and the proportionality coefficient between the carrier density and the gate voltage $\left(n=\beta V_{\mathrm{g}}{ }^{\prime}\right)$, respectively.

Figures 4(a) and 4(b) show the longitudinal $\left(\sigma_{\mathrm{xx}}\right)$ and Hall $\left(\sigma_{\mathrm{xy}}\right)$ conductivities, respectively. The expected Hall plateaus corresponding to $\nu= \pm 2$ are clearly observed. However, we cannot see distinct plateaus corresponding to $\nu= \pm 6$ due to disorder broadening of the Landau levels at 
higher energy. Interestingly, an unexpected dip and unusual Hall plateau appear at $\nu=0$ in $\sigma_{\mathrm{xx}}$ and $\sigma_{\mathrm{xy}}$, respectively. This becomes more pronounced with decreasing temperature, which indicates the insulating $\nu=0$ quantum Hall state. We believe that it is due to a gap formation at the Dirac point resulting in slight splitting of the central LL at $\nu=0$. In fact, the insulating $\nu=0$ quantum Hall state has been experimentally observed ${ }^{20-22}$ its origin is still controversial. Possible explanations include lifting of LL due to a spin-valley symmetry breaking, ${ }^{20}$ counter-propagating edge states,${ }^{21}$ and gap opening in the magnetic field. ${ }^{22}$ All these previous experiments were done using relatively high quality samples made of exfoliated graphene. We suppose that the appearance of the insulating $\nu=0$ quantum Hall state in our case is most likely due to the disorder induced gap opening. ${ }^{9,23} \mathrm{We}$ found that the insulating $\nu=0$ quantum Hall state disappears when the relatively high quality $\left(\mu \approx 3000 \mathrm{~cm}^{2} / \mathrm{Vs}\right) \mathrm{CVD}$-graphene was measured.

Figure 4(c) shows the odd-function component of magneto-TEP $\left(S_{\mathrm{xx}}{ }^{\text {odd }}\right)$ at various temperatures. The overall magnitude of the oscillations tends to decrease with lowering temperature except for the peaks near $\nu=|4|$, which start to increase at $T=30 \mathrm{~K}$. Here, the two peaks signify the LL development at $\nu=|4|$. At $T=5 \mathrm{~K}$, interestingly, $S_{\mathrm{xx}}$ odd shows large fluctuations near the Dirac point $\left(V_{\mathrm{g}}{ }^{\prime} \approx 0\right)$ in the inset. This is probably due to dominating effect of the band gap opening at lower temperature. Theoretically, it has earlier been reported that the "gapped" graphene should show anomalously large bump near the gap. ${ }^{24}$ An enhanced TEP $\left(\approx 10^{2} \mu \mathrm{V} / \mathrm{K}\right)$ was indeed observed by experiments; gapped
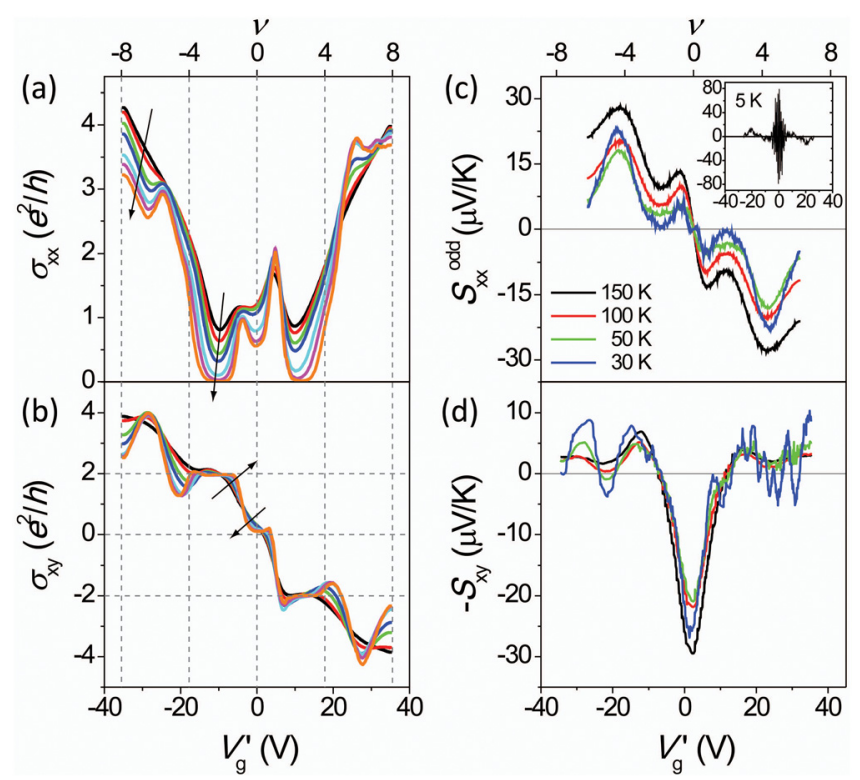

FIG. 4. (a) The longitudinal conductivity $\sigma_{\mathrm{xx}}$ and (b) Hall conductivity $\sigma_{\mathrm{xy}}$ as functions of the shifted gate voltage $V_{\mathrm{g}}{ }^{\prime}\left(=V_{\mathrm{g}}-V_{\mathrm{DP}}\right)$ for $T=150,100,50$, $30,10,5$, and $2 \mathrm{~K}$ in the magnetic field $B=13 \mathrm{~T}$. The arrows indicate the changes corresponding to lowering temperature. The vertical dashed lines represent filling factors corresponding to $\nu=0, \pm 4, \pm 8$. (c) The odd-function component of magneto-TEP $\left(S_{\mathrm{xx}}{ }^{\text {odd }}\right)$ and (d) Nernst signal $\left(S_{\mathrm{xy}}\right)$ for $T=150,100,50$, and $30 \mathrm{~K}$ at $B=13 \mathrm{~T}$. The inset in (c) displays $S_{\mathrm{xx}}{ }^{\text {odd }}$ at temperatures of $5 \mathrm{~K}$. Large fluctuations are observed near the Dirac point. (d) $S_{\mathrm{xy}}$ shows distinct electron-hole asymmetry with decreasing temperature. Especially fluctuations are seen upon decreasing temperature on the electron side of the $V_{\mathrm{g}}{ }^{\prime}$-scale. bilayer graphene under the perpendicular electric field ${ }^{25}$ and gapped multilayer graphene by oxygen plasma induced disorder. ${ }^{9}$ The large TEP fluctuations in our case could be caused by the disorder associated with inhomogeneity of our sample. Meanwhile, $S_{\mathrm{xx}}{ }^{\text {odd }}$ at $T=5 \mathrm{~K}$ preserves ordinary peaks lying near $\nu=|4|$ although their values $(\approx 13 \mu \mathrm{V} / \mathrm{K})$ are smaller than the theoretically expected $\left(k_{\mathrm{B}} / e\right) \ln 2 \approx 59.7 \mu \mathrm{V} / \mathrm{K}$.

Finally, we show the Nernst signal $S_{\mathrm{xy}}$ for various temperatures (see Figure 4(d)). The signatures of LL come from $S_{\mathrm{xy}}$ where $d^{2} S_{\mathrm{xy}} / d V_{\mathrm{g}}{ }^{2} \approx 0$ near $\nu=|4|$. In particular, the electron-hole asymmetry increases with decreasing temperature and fluctuations become seen only on the electron side $\left(V_{\mathrm{g}}{ }^{\prime} \geq 20\right)$. We attribute this to sensitivity of $S_{\mathrm{xy}}$ to sample the inhomogeneity and presence of impurities, ${ }^{26}$ which requires further study.

We have measured TEP of inhomogeneous CVDgraphene. The significant electron-hole point asymmetry in the TEP measurements can be attributed to individual graphene regions having different TEPs in the inhomogeneous CVD-graphene. At high magnetic field and low temperature, we observe anomalously large fluctuations in $S_{\mathrm{xx}}$ near the Dirac point. This could be accounted for by the insulating $\nu=0$ quantum Hall state resulting from the disorder-induced energy gap opening around Dirac point. On the other hand, Nernst signal $\left(S_{\mathrm{xy}}\right)$ shows fluctuations only for electron-like carriers. Our TEP measurements reveal additional characteristics of CVD-graphene, which are difficult to obtain from the measurement of resistance alone.

Financial support from the Swedish Research Council, the Swedish Foundation for Strategic Research (Sweden), and the Leading Foreign Research Institute Recruitment Program (0409-20100156) of National Research Foundation and the FPRD of BK21 through the Ministry of Education, Science and Technology, Korea is greatly appreciated. Clean-room processing has been achieved using equipment sponsored by the Knut and Alice Wallenberg Foundation.

${ }^{1}$ S. Das Sarma, S. Adam, E. H. Hwang, and E. Rossi, Rev. Mod. Phys. 83(2), 407 (2011).

${ }^{2}$ T. Löfwander and M. Fogelström, Phys. Rev. B 76(19), 193401 (2007); E. H. Hwang, E. Rossi, and S. Das Sarma, ibid. 80(23), 235415 (2009); L. Zhu, R. Ma, L. Sheng, M. Liu, and D.-N. Sheng, Phys. Rev. Lett. 104(7), 076804 (2010).

${ }^{3}$ Y. M. Zuev, W. Chang, and P. Kim, Phys. Rev. Lett. 102(9), 096807 (2009); P. Wei, W. Bao, Y. Pu, C. N. Lau, and J. Shi, ibid. 102(16), 166808 (2009).

${ }^{4}$ J. G. Checkelsky and N. P. Ong, Phys. Rev. B 80(8), 081413 (2009).

${ }^{5}$ D. Wang and J. Shi, Phys. Rev. B 83(11), 113403 (2011).

${ }^{6}$ X. Wu, Y. Hu, M. Ruan, N. K. Madiomanana, C. Berger, and W. A. de Heer, Appl. Phys. Lett. 99(13), 133102 (2011).

${ }^{7}$ J. Yin, J. Zhou, X. Li, Y. Chen, G. Tai, and W. Guo, Appl. Phys. Lett. 99(7), 073103 (2011).

${ }^{8}$ A. N. Sidorov, A. Sherehiy, R. Jayasinghe, R. Stallard, D. K. Benjamin, Q. Yu, Z. Liu, W. Wu, H. Cao, Y. P. Chen, Z. Jiang, and G. U. Sumanasekera, Appl. Phys. Lett. 99(1), 013115 (2011).

${ }^{9}$ N. Xiao, X. Dong, L. Song, D. Liu, Y. Tay, S. Wu, L.-J. Li, Y. Zhao, T. Yu, H. Zhang, W. Huang, H. H. Hng, P. M. Ajayan, and Q. Yan, ACS Nano 5(4), 2749 (2011).

${ }^{10}$ A. V. Babichev, V. E. Gasumyants, and V. Y. Butko, J. Appl. Phys. 113(7), 076101 (2013).

${ }^{11}$ S. Jie, N. Lindvall, M. T. Cole, K. T. T. Angel, W. Teng, K. B. K. Teo, D. Chua, J. Liu, and A. Yurgens, IEEE Trans. Nanotechnol. 11(2), 255 (2012). 
${ }^{12}$ J. P. Small, L. Shi, and P. Kim, Solid State Commun. 127(2), 181 (2003).

${ }^{13}$ Q. Yu, L. A. Jauregui, W. Wu, R. Colby, J. Tian, Z. Su, H. Cao, Z. Liu, D. Pandey, D. Wei, T. F. Chung, P. Peng, N. P. Guisinger, E. A. Stach, J. Bao, S.-S. Pei, and Y. P. Chen, Nature Mater. 10(6), 443 (2011).

${ }^{14}$ D. C. Elias, R. R. Nair, T. M. G. Mohiuddin, S. V. Morozov, P. Blake, M. P. Halsall, A. C. Ferrari, D. W. Boukhvalov, M. I. Katsnelson, A. K. Geim, and K. S. Novoselov, Science 323(5914), 610 (2009).

${ }^{15}$ A. Eckmann, A. Felten, A. Mishchenko, L. Britnell, R. Krupke, K. S. Novoselov, and C. Casiraghi, Nano Lett. 12(8), 3925 (2012).

${ }^{16}$ J.-H. Chen, W. G. Cullen, C. Jang, M. S. Fuhrer, and E. D. Williams, Phys. Rev. Lett. 102(23), 236805 (2009).

${ }^{17}$ J. Heo, H. J. Chung, S.-H. Lee, H. Yang, D. H. Seo, J. K. Shin, U. I. Chung, S. Seo, E. H. Hwang, and S. Das Sarma, Phys. Rev. B 84(3), 035421 (2011).

${ }^{18}$ A. B. Kaiser, C. Gómez-Navarro, R. S. Sundaram, M. Burghard, and K. Kern, Nano Lett. 9(5), 1787 (2009).

${ }^{19}$ S. Cho, S. D. Kang, W. Kim, E.-S. Lee, S.-J. Woo, K.-J. Kong, I. Kim, H.D. Kim, T. Zhang, J. A. Stroscio, Y.-H. Kim, and H.-K. Lyeo, Nature Mater. 12(10), 913 (2013).
${ }^{20}$ Y. Zhang, Z. Jiang, J. P. Small, M. S. Purewal, Y. W. Tan, M. Fazlollahi, J. D. Chudow, J. A. Jaszczak, H. L. Stormer, and P. Kim, Phys. Rev. Lett. 96(13), 136806 (2006); Y. Zhao, P. Cadden-Zimansky, F. Ghahari, and P. Kim, ibid. 108(10), 106804 (2012).

${ }^{21}$ D. A. Abanin, K. S. Novoselov, U. Zeitler, P. A. Lee, A. K. Geim, and L. S. Levitov, Phys. Rev. Lett. 98(19), 196806 (2007).

${ }^{22}$ J. G. Checkelsky, L. Li, and N. P. Ong, Phys. Rev. Lett. 100(20), 206801 (2008); L. Zhang, Y. Zhang, M. Khodas, T. Valla, and I. A. Zaliznyak, ibid. 105(4), 046804 (2010).

${ }^{23}$ J. Bai, X. Zhong, S. Jiang, Y. Huang, and X. Duan, Nat. Nanotechnol. 5(3), 190 (2010); C.-K. Yang, Carbon 48(13), 3901 (2010); B. S. Pujari and D. G. Kanhere, J. Phys. Chem. C 113(50), 21063 (2009); N. Amirhasan, C. Mirco, V. Tom, P. Geoffrey, C. Francesca, H. v. d. V. Marleen, H. Johan, M. H. Marc, G. Stefan De, and F. S. Bert, Nanotechnology 21(43), 435203 (2010).

${ }^{24}$ S. G. Sharapov and A. A. Varlamov, Phys. Rev. B 86(3), 035430 (2012); A. A. Patel and S. Mukerjee, ibid. 86(7), 075411 (2012).

${ }^{25}$ C.-R. Wang, W.-S. Lu, L. Hao, W.-L. Lee, T.-K. Lee, F. Lin, I. C. Cheng, and J.-Z. Chen, Phys. Rev. Lett. 107(18), 186602 (2011).

${ }^{26}$ S.-P. Chao and V. Aji, Phys. Rev. B 84(15), 155430 (2011). 